\title{
Obesity and COVID-19: Take Two-Has Our Saw Been Adequately Sharpened?
}

\author{
Marvin Wei Jie Chua ${ }^{1}$ (D)
}

Received: 18 May 2021 / Revised: 25 May 2021 / Accepted: 26 May 2021 / Published online: 9 June 2021

(C) The Author(s), under exclusive licence to Springer Science+Business Media, LLC, part of Springer Nature 2021

$31^{\text {st }}$ December 2019: a date which will undoubtedly be etched in our memories as the first time the world became aware of the severe acute respiratory syndrome coronavirus 2 (SARSCoV-2) virus. The rest, as they say, is history. The first case of COVID-19 hit our shores on $23^{\text {rd }}$ January 2020, with an alarming increase in community transmission prompting the institution of a "circuit breaker" in April 2020, which included stay-home measures, telecommuting and closure of schools, not dissimilar to the more common term "lockdown". At that time, I observed and reported the worrisome bidirectional relationship between COVID-19 and obesity, which indeed reinforce each other in an inexorable vicious cycle [1,2]. Obesity increases the risk of COVID-19, including severe COVID-19 and death [1]. At the same time, implementation of the aforementioned measures to curb virus transmission led to increased consumption of processed, high-caloric food and an increasingly sedentary lifestyle [3], all of which threatened to lead to sky-rocketing rates of obesity and its associated complications in the "clash of two pandemics" [1].

However, it was precisely at that time when medical attention and services for patients with obesity were most urgently needed, that they seemed to be the most lacking. Nonessential clinic appointments including weight management services and bariatric surgeries were postponed to conserve resources and reduce the risk of viral transmission. Many patients also elected not to turn up for their clinic appointments due to fear of infection. Recognizing the need for physicians to "sharpen the saw" in view of this "new normal", I had described methods to rebuild our clinical pathways for the future to manage the post-COVID-19 fallout, including telemedicine, motivational interviewing and mindfulness [2].

Marvin Wei Jie Chua

marvin.chua.w.j@singhealth.com.sg

1 Endocrinology Service, Department of General Medicine, Sengkang General Hospital, 110 Sengkang East Way,

544886 Singapore, Singapore
By December 2020, community transmission rates had fallen to a sufficiently low rate that a flicker of light at the end of a long tunnel was in sight. Alas, recent new clusters of infection sprung up in May 2021, prompting the swift reintroduction of restrictions including stay-home measures, reduction in size of group gatherings and prohibition of dining-in. Similar to the situation 1 year ago, elective surgeries and non-essential clinic appointments were postponed. The same clinics which were bustling 1 month ago were now near-empty, likely contributed by increased public fears due to a recent cluster arising within an acute hospital.

Many countries would be no stranger to this "second coming", with repeated widespread waves of infections after initial apparent control. That said, as an Endocrinologist managing patients with obesity who was seemingly in the "building back" phase following the initial wave of COVID-19, only to be pushed back into the "ravine", I had a few reflections which might be useful for fellow physicians.

Firstly, it appears that there are a growing number of COVID-19 cases in children. It was previously established that children are at lower risk of viral infection and transmission $[4,5]$. However, based on the cases to date locally, it appears that a significant number of children have been affected, with a number of clusters being linked to schools and enrichment centres. Children may also be at increased risk of long-COVID, a debilitating post-COVID illness [6], and to date, there is no approved vaccine for young children. At the same time, childhood obesity is a burgeoning problem. In a health survey done in Singapore in 2017, 13\% of school children and $10 \%$ of 5 -year-old children were overweightmost concerningly, $70 \%$ of children who were overweight at the age of 7 remained overweight as adults. Therefore, the "clash of two pandemics" clearly starts from childhood and intensifies throughout the lifespan. An interesting perspective reported by De Rubeis et al. is that disasters, including pandemics, might have long-term adverse effects on cardiometabolic health which may only be evident later in life [7]. We need to urgently take action to curb the growing rates of 
childhood obesity and focus our efforts on preventive measures to try to "nip the problem in the bud" and decrease the burden of upstream problems.

Although the importance of tackling obesity from childhood is undisputed, it clearly involves large-scale changes on a societal, national or indeed global level. While this is ongoing, in our capacity as individual physicians, is there an immediate difference we can make to our patients in the current climate?

Telemedicine is a powerful tool that allows continued delivery of healthcare services while circumventing the aforementioned concerns of virus transmission [8,9]. A systematic review in children with obesity found that compared to faceto-face consultation, telemedicine led to similar outcomes including body mass index (BMI), nutrition, physical activity and satisfaction scores. However, telemedicine is not without its downsides [9]. Ensuring privacy and data security is of utmost importance to maintain patient confidentiality. Anthropometric and laboratory data, which are often essential in making further treatment decisions, may not be readily obtained.

The key question is, can telemedicine truly replace face-toface consultations? The management of patients with obesity requires an emphasis on not just physical, but also psychological and social health-consistent with the World Health Organization (WHO) definition of "health", as obesity is associated with increased psycho-social distress. This in turn relies heavily on the patient-physician relationship - one that is based on trust and requires consistent effort and time to nurture. In a study, physicians were found to have lower levels of emotional rapport during consultation with overweight and obese patients, which may diminish adherence to recommendations and decrease the effectiveness of behavioural change counselling [10]. Therefore, the importance of forging a strong patient-physician relationship cannot be overstated, but how can we effectively do so from behind a screen? This is where two tools which I had earlier describedmotivational interviewing and mindfulness, may be applied [2]. With motivational interviewing, we "walk the path" with the patient regardless of the state of readiness for behavioural change, giving the freedom to change on his/her own terms while providing constant support [2]. In a mindful state, we may be able to detect subtle changes in a patient's body language which might otherwise be easily missed over a virtual platform, especially if we are intent on pushing forward the agenda of our consultation [2].

Nonetheless, telemedicine remains an effective tool in our armamentarium in these times. Physicians should focus on "sharpening the saw", with the goal of delivering the same, if not better care through telemedicine. On a larger scale, perhaps a "hybrid" model, where teleconsultation is default while face-to-face consultation is reserved for initial consultation and patients with significant psycho-social issues, could be a model of care delivery in the future.

In conclusion, we physicians managing patients with obesity have an uphill task ahead - when will the pandemic truly end? Only time will tell. Until then, I present two proposed changes which are complementary to each other-one on a large scale, societal level to focus our efforts on tackling childhood obesity, and the other on a one-to-one, patient-physician level to deliver the best possible care through telemedicine.

\section{Declarations}

Human and animal rights This article does not contain any studies with human participants or animals performed by the author.

Informed consent Informed consent does not apply, as this article does not include data or description of any individual patient.

Conflict of interest The author declares that there is no conflict of interest.

\section{References}

1. Chua MWJ, Zheng S. Obesity and COVID-19: the clash of two pandemics. Obes Res Clin Pract. 2020;14:380-2. https://doi.org/10. 1016/j.orcp.2020.06.003.

2. Chua MWJ. Managing patients with obesity in the post COVID-19 world: time to sharpen the saw. Obes Res Clin Pract. 2021;15:85-8.

3. Chew HSJ, Lopez V. Global impact of COVID-19 on weight and weight-related behaviors in the adult population: a scoping review. Int J Environ Res Public Health. 2021;18

4. Yung CF, Kam KQ, Chong CY, et al. Household transmission of severe acute respiratory syndrome coronavirus 2 from adults to children. J Pediatr. 2020;225:249-51.

5. Yung CF, Kam KQ, Nadua KD, et al. Novel coronavirus 2019 transmission risk in educational settings. Clin Infect Dis Off Publ Infect Dis Soc Am. 2021;72:1055-8.

6. Thomson H. Children with long covid. New Sci. 1971;2021(249): $10-1$.

7. De Rubeis V et al. Impact of disasters, including pandemics, on cardiometabolic outcomes across the life-course: a systematic review. BMJ Open. 2021;11:e047152.

8. Umano GR, di Sessa A, Guarino S, et al. Telemedicine in the COVID-19 era: taking care of children with obesity and diabetes mellitus. World J Diabetes. 2021;12:651-7.

9. Whitley A, Yahia N. Efficacy of clinic-based telehealth vs. face-toface interventions for obesity treatment in children and adolescents in the United States and Canada: a systematic review. Child Obes Chi. 2021;2020:0347. https://doi.org/10.1089/chi.2020.0347.

10. Gudzune KA, Beach MC, Roter DL, et al. Physicians build less rapport with obese patients. Obes Silver Spring Md. 2013;21:214652.

Publisher's Note Springer Nature remains neutral with regard to jurisdictional claims in published maps and institutional affiliations. 